Résumés des conférences et travaux

\title{
Langue et sources documentaires coptes
}

\section{Alain Delattre}

\section{OpenEdition \\ Journals}

Édition électronique

URL : https://journals.openedition.org/ashp/3662

DOI : $10.4000 /$ ashp.3662

ISSN : 1969-6310

Éditeur

Publications de l'École Pratique des Hautes Études

\section{Édition imprimée}

Date de publication : 1 septembre 2020

Pagination : 113-115

ISSN : 0766-0677

Référence électronique

Alain Delattre, "Langue et sources documentaires coptes ", Annuaire de l'École pratique des hautes études (EPHE), Section des sciences historiques et philologiques [En ligne], 151 | 2020, mis en ligne le 09 juillet 2020, consulté le 06 juillet 2021. URL : http://journals.openedition.org/ashp/3662 ; DOI : https:// doi.org/10.4000/ashp.3662 


\title{
LANGUE ET SOURCES DOCUMENTAIRES COPTES
}

\author{
Directeur d'études : M. Alain Delattre
}

Programme de l'année 2018-2019: I. L'épigraphie funéraire chrétienne d'Égypte et de Nubie. — II. Documents juridiques de Moyenne-Égypte (suite).

L'épigraphie funéraire chrétienne d'Égypte et de Nubie connaît un regain d'intérêt depuis plusieurs années, comme en témoignent la vaste synthèse rédigée par B. Tudor en 2011 et les travaux récents de J. van der Vliet ${ }^{1}$. Coptes ou grecques, les épitaphes de l'Antiquité tardive et du début de l'époque médiévale ont été publiées par centaines et les inédits restent nombreux. La documentation est caractérisée par la vivacité des traditions locales et doit s'interpréter, autant que possible, dans son environnement physique (archéologique) et cultuel (liturgique), puisque chaque texte constitue, pour les vivants, un résumé et une amorce du rituel de commémoration du défunt. Dans la conférence de l'année 2018-2019, trois sites ont été étudiés en profondeur : les monastères de Saqqarah et de Baouit et la ville d'Antinoupolis.

Les textes épigraphiques du monastère d'apa Jérémias à Saqqarah ont été édités par H. Thompson dans les rapports de fouilles de J. Quibell ${ }^{2}$. Quatre cents documents ont été publiés, notamment des inscriptions funéraires découvertes dans les salles 1823 et $1772 \mathrm{~S}$, de vastes pièces destinées à l'inhumation de personnages importants de la communauté et aux commémoraisons funéraires. Plusieurs séances ont été consacrées à la lecture et à la datation des épitaphes. Ainsi, la formule de datation de la pierre tombale du secrétaire Apakuré (Quibell, III $11=$ Wietheger 86), qui associe le comput des martyrs, l'indiction et l'année hégirienne, a été lue, 1. 7-10:

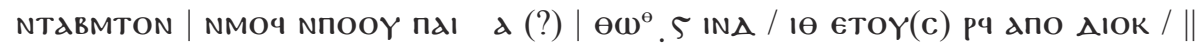
$\phi \kappa r$; l'éditeur expliquait en note sa perplexité face au texte. L'examen de l'image de la pierre a permis de remettre la date sur pied et de lire : NTABMTON | NMO4 NחOOY

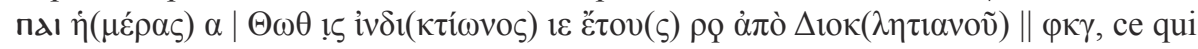
peut se traduire «Il s'est reposé aujourd'hui, c'est-à-dire le dimanche 16 Thôth de la $15^{\mathrm{e}}$ indiction, soit l'année 190 (de l'hégire) et 523 à partir de Dioclétien », ce qui correspond au 13 septembre 806; les différents calendriers sont parfaitement cohérents

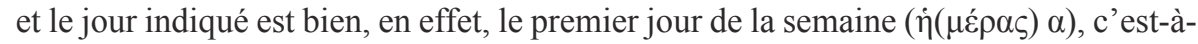
dire un dimanche. D'autres formules de datation ont pu être rétablies, éliminant ainsi plusieurs problèmes de chronologie soulevés par H. Thompson. On notera enfin que le terme $\mathbf{x} \times n$, littéralement « l'heure », utilisé aux Kellia pour désigner l'année indictionnelle, comme l'a montré P. Luisier ${ }^{3}$, est attesté dans le même sens à Saqqarah.

1. Bianca Tudor, Christian Funerary Stelae of the Byzantine and Arab Periods from Egypt, Marbourg, 2011; Jacques van der Vliet, The Christian Epigraphy of Egypt and Nubia, éd. Renate Dekker, Abingdon, New York, 2018.

2. James E. Quibell, Excavations at Saqqara [II], 1906-1907 ; [III], 1907-1908; [IV], 1908-1909, 1909 1910, Le Caire, 1908, 1909, 1912; voir aussi la synthèse de Cäcilia Wietheger, Das Jeremias-Kloster zu Saqqara unter besonderer Berücksichtigung der Inschriften, Altenberge, 1992.

3. Philippe Luisier, «Les années de l'indiction dans les inscriptions des Kellia », ZPE, 159 (2007), p. 217 222. 
L'épigraphie funéraire d'Antinoupolis est particulièrement riche : des dizaines de textes grecs et coptes sont publiés et plus de deux cents inscriptions coptes sont encore inédites. La plupart des documents proviennent de la nécropole nord du site et datent des $\mathrm{VI}^{\mathrm{e}}-\mathrm{VIII}^{\mathrm{e}}$ siècles. Beaucoup d'épitaphes invoquent le saint patron de la ville, Kollouthos, mais le corpus se signale par la variété des formulaires utilisés. L'accent a été mis en particulier sur les textes de lamentations (Totenklage) qui déplorent, dans un style poétique, la nature fugace et éphémère de la vie et le caractère cruel et inexorable de la mort.

Le monastère de Baouît enfin a livré une abondante moisson d'inscriptions, publiées dans les rapports de fouilles ${ }^{4}$. Les éditions de J. Clédat, ou pour mieux dire ses transcriptions brutes, puisque les coupes de mots ne sont pas indiquées et que les textes ne sont pas commentés, sont souvent imparfaites et peuvent parfois être améliorées. La salle désignée sous le nom de chapelle III abrite ainsi diverses inscriptions funéraires commémoratives peintes sur les murs. L'une d'entre elles ( ${ }^{\circ}$ IV) invoque les moines «qui se sont couchés dans le Seigneur », c'est-à-dire qui sont décédés, et des moines vivants qui leur rendent hommage; les noms de plusieurs d'entre eux peuvent être restitués, par exemple l'archimandrite apa Joa(nnês) et papa Mercure

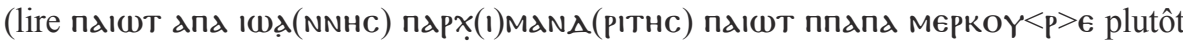

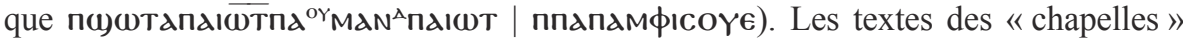
VII, XVII et LIV ont ensuite été examinés, en particulier les inscriptions funéraires de moines décédés à la fin des années 730 (chapelle XVII, ${ }^{\text {os }}$ IV, V et IX). Signalons enfin que l'étude des graffiti de la chapelle VII a permis d'identifier quatre citations des Psaumes, non reconnues jusqu'à présent, auxquelles on a pu ajouter deux textes psalmiques de Saqqarah, dont un avait également échappé à l'attention du monde savant ${ }^{5}$.

La seconde partie de la conférence a été consacrée aux documents juridiques de Moyenne-Égypte. Nous avons d'abord examiné un papyrus exceptionnel de la Staatsund Universitätsbibliothek de Hambourg, qui conserve un contrat de location de droit de pêche. Ce document constitue un unicum en copte et les textes de ce type sont rares même en grec : neuf exemplaires à peine sont publiés, dont un seul de l'époque byzantine. Notre contrat a été écrit par une communauté monastique d'Assiout ou de ses environs et le bailleur est un comte du nom d'Anastase; la location porte sur le droit de pêche dans un réservoir et l'institution religieuse s'engage à fournir du poisson frais chaque jour et une certaine quantité de salaisons à l'année ${ }^{6}$. D'autres documents relatifs à la pêche ont ensuite été examinés, notamment P. Soc. Arch. Copt. Inv. $7^{7}$, dont l'édition a pu être améliorée.

Deux papyrus de la collection de la Papyrussammlung ont été présentés ensuite. Le premier, $C P R$ IV 93, est adressé au dikaion, c'est-à-dire à la personnalité juridique, d'une institution religieuse de la ville d'Hermoupolis. Il affecte la forme d'un

4. En particulier, Jean Clédat, Le monastère et la nécropole de Baouît, t. I, Le Caire, 1904.

5. Alain Delattre, «Des citations des Psaumes dans les inscriptions des monastères de Saqqarah et Baouît », Chronique d'Égypte, 94 (2019), p. 181-187.

6. Le document est désormais publié, cf. Alain Delattre et Naïm Vanthieghem, « Un contrat de location de droit de pêche (P. Hamb. Copt. Inv. 10) », Journal of Coptic Studies, 21 (2019), p. 19-27.

7. Leslie S. B. MacCoull, «Coptic Documentary Papyri in the Collection of the Society for Coptic Archaeology, Cairo », dans Atti del XVII Congresso Internazionale di Papirologia, II, Naples, 1984, p. $777-785$. 
contrat de prêt, annulé par de grandes lignes qui traversent le texte. Il constitue un bon exemple de la variété des documents de ce genre et surtout de l'usage qui peut en être fait pour formaliser divers types de relations contractuelles. Le contenu du second, $C P R$ IV 197, est plus difficile à caractériser; l'éditeur, W. C. Till, l'a classé parmi les documents de nature incertaine. La paléographie permet de le dater du $\mathrm{IX}^{\mathrm{e}}$ siècle, peut-être de l'année 866, si la lecture de la ligne 9 est correcte; la provenance est en revanche difficile à déterminer. Le texte est écrit par sept personnes, dont les identités sont déclinées aux premières lignes et qui ont toutes confirmé leur accord à la fin du papyrus, le plus souvent de leur propre main. Le document est adressé à un officiel musulman du nom de Ḥasan, fils de 'Abd al-raḥmān qui, comme l'a vu L. Berkes ${ }^{8}$, porte le titre de al-qustal, c'est-à-dire de receveur. Le texte semble annoncer le paiement de trois sous d'or dans le cadre d'un redressement fiscal (al-istidrāk).

Divers papyrus juridiques coptes de la Sorbonne ont été présentés ensuite, qui illustrent différents types documentaires : des contrats de location ou de prêt, des garanties, etc.

La séance du 15 mars a eu lieu à l'Institut de papyrologie de la Sorbonne, où étaient réunis pour une semaine les membres de l'équipe en charge de l'étude des archives du pagarque d'Edfou Papas, en particulier de son versant copte. Deux pièces des archives, qui se trouvent en dépôt à la Sorbonne, ont été examinées à cette occasion.

Signalons enfin que trois jeunes chercheurs ont été invités à présenter leurs recherches au cours de l'année 2018-2019. Les 7 et 14 décembre, $\mathrm{M}^{\mathrm{me}}$ Eline Scheerlinck a fait part de son travail sur le rôle social des élites locales au début de la période islamique, en particulier dans la gestion des fugitifs; elle a notamment mis en évidence les divers éléments de la procédure d'obtention des documents 入огос M̄nnoyte. Le 18 janvier 2019, M. Naïm Vanthieghem a exposé ses recherches sur le dossier trilingue (grec, copte et arabe) de Kosma, fils de Prôou, moine à Saqqarah dans les années 720-730. M $^{\text {me }}$ Élodie Mazy a présenté le 22 mars 2019 son étude de l'inédit P. Sorb. Inv. 2276, qui porte des comptes, en grec au recto et en copte au verso. Le document provient de Baouît et date du vIII ${ }^{\mathrm{e}}$ siècle; il donne un aperçu inédit sur la comptabilité et plus largement sur la vie économique de ce grand monastère de Moyenne-Égypte.

8. Une réédition commentée du document est en préparation par Lajos Berkes, Naïm Vanthieghem et moimême. 